\title{
EXPANSION ASPECT OF COLOR TRANSPARENCY ON THE LATTICE
}

\author{
D. Makovoz and G. A. Miller
}

November 23, 2018

Department of Physics, FM-15, University of Washington, Seattle, WA 98195, USA

\begin{abstract}
The opportunity to observe color transparency (CT) is determined by how rapidly a small-sized hadronic wave packet expands. Here we use SU(2) lattice gauge theory with Wilson fermions in the quenched approximation to investigate the expansion. The wave packet is modeled by a point hadronic source, often used as an interpolating field in lattice calculations. The procedure is to determine the Euclidean time (t), pion channel, Bethe-Salpeter amplitude $\Psi(r, t)$, and then evaluate $b^{2}(t)=\int d^{3} r \Psi(r, t) r^{2} \sin ^{2} \theta \Psi_{\pi}(r)$. This quantity represents the soft interaction of a small-sized wave packet with a pion. The time dependence of $b^{2}(t)$ is fit as a superposition of three states, which is found sufficient to reproduce a reduced size wave packet. Using this superposition allows us to make the analytic continuation required to study the wave packet expansion in real time. We find that the matrix elements of the soft interaction $\hat{b}^{2}$ between the excited and ground state decrease rapidly with the energy of the excited state.
\end{abstract}




\section{INTRODUCTION}

Color transparency (CT) can be defined as the absence of or reduced final state interaction of a nucleon with the nucleus in semiexclusive high momentum transfer processes. Examples are the (e,e'p) and (p,pp) reactions occuring on nuclear targets, in which the detected protons have enough energy to ensure that no pions are produced. For color transparency to be observed three conditions have to be met [1].

(i) A small wave packet is formed in a high momentum transfer reaction. This wave packet is sometimes dubbed a point like configurations(PLC).

(ii) A small wave packet interacts weakly with the nucleus.

(iii) The wave packet escapes the nucleus before expanding.

These conditions are true in the perturbative regime [2, 3], but have to be tested in the nonperturbative regime. Lattice QCD is a natural tool to use in this investigation. It can be used to investigate the form of the hadron-hadron interaction [4], to probe the wave packet (hopefully PLC) produced in a high momentum transfer reaction, and to examine the expansion of the PLC.

The separation of the interaction into the hard high momentum transfer part and the soft final state interaction part is crucial for CT. To be specific consider (e,e'p) reaction. In this case the hard process is the absorption of the virtual photon by a nucleon in the nucleus. An additional hard interaction is expected to be suppressed by the experimental kinematics, and the final state interaction of the PLC with the nucleus is of a soft lowmomentum-transfer nature. There are two amplitudes to be compared. The first one is for the proton to escape the nucleus without interaction. The second one is for the proton to be detected after the PLC is scattered by the soft interaction with the nuclear medium. CT can be obtained if the ratio of the second amplitude to the first one vanishes in the limit of $Q \rightarrow \infty$.

Ideally one would like to reconstruct on the lattice the process described above: to create a wave packet by acting with the electromagnetic current operator on the proton, and to evaluate the amplitude of this wave packet to be transformed into a proton by 
some realistic nucleon-PLC interaction. In this paper we will not take on this challenging and complex problem. We concentrate on one aspect of the question - the expansion of a model small size wave packet. To further simplify our task we consider two-quark pion instead of three-quark proton.

We start by defining the necessary lattice constructions in Sec.2. A point source, one of the conventionally used interpolating fields, is used to generate the small size wave packet - PLC. A pion is detected in the final state after the PLC experiences the soft interaction. A model form of the soft interaction $\widehat{b}^{2}$ [5] is used, with $b$ representing the transverse separation of the quarks. The wave packet undergoes Euclidean time evolution. The quantity $b^{2}(t)$, which measures the strength of the soft interaction of the evolving PLC with the nuclear medium, is calculated as a function of the Euclidean time $t$. The analytical continuation to the Minkowsky time $\tau$ is then performed. In Sec.3 the lattice details are given. The purpose of using lattice QCD is to obtain matrix elements from the first principle calculations. Unfortunately it entails the Euclidean time evolution. Our procedure is to treat the wave packet as a coherent sum of physical states, so the real problem is how to detect as many states as possible before they decay away. These states are extracted by a many pole fit of the lattice results. This procedure is complicated and controversial. We discuss it for the two-point correlation function in Sec.4. To perform the analytical continuation the $b^{2}(t)$ is fit with the sum of three exponentials. The details of the $b^{2}(t)$ three pole fit are given in Sec.5. The results of the analytical continuation and their discussion are presented in Sec.6. The relative merits of using a full or a diagonal covariance matrix to fit observables is discussed in the Appendix.

\section{FORMALISM}

We want to consider the expansion of a small size wave packet. To form such a wave packet we take advantage of the point interpolating field $\phi(\mathbf{x}, t)$, which has the following 
form in the pseudoscalar channel

$$
\phi(\mathbf{x}, t)=d(\mathbf{x}, t) \gamma_{5} \bar{u}(\mathbf{x}, t)
$$

By acting on the vacuum $\phi(\mathbf{x}, t)$ creates an object similar to a PLC, since the quark and the antiquark are created at the same site. This wave packet can be expanded in a complete set of zero momentum states $\mid \nu>$ :

$$
\sum_{\mathbf{x}} \phi(\mathbf{x}, 0)\left|0>=>\sum_{\nu}\left(\int d \nu\right) c_{\nu}\right| \nu>\equiv \mid P L C>
$$

where the summation over $\mathbf{x}$ projects out the zero momentum states. The coefficient $c_{\nu}$,

$$
c_{\nu}=<\nu|\phi(0,0)| 0>/ \sqrt{2 E_{\nu}}
$$

is the strength with which the state $\mid \nu>$ is excited from the vacuum, $\sqrt{2 E_{\nu}}$ is a normalization factor. We have to note that even though the interpolating field creates a quark and an antiquark at the same site, there is a non-zero probability to detect them separated by some distance at the same time they are created. This occurs because fermion propagators do not vanish outside of the light cone.

If we transport the $\mid P L C>$ with the Euclidean transfer matrix $\exp (-\widehat{H} t)$ and then contract it with itself we obtain the two-point correlation function $G_{2}(t)$ :

$$
\begin{array}{r}
G_{2}(t)=\sum_{\mathbf{x}}<0|\phi(\mathbf{x}, t) \phi(0,0)| 0>= \\
=\sum_{\nu}\left(\int d \nu\right)\left|c_{\nu}\right|^{2} e^{-E_{\nu} t}
\end{array}
$$

$G_{2}(t)$ plays a complementary role in our investigation, as will be seen below.

Our main objective is to evaluate the strength of the soft interaction of the expanding PLC with the nucleus. This interaction can be described ([5] and references therein) by

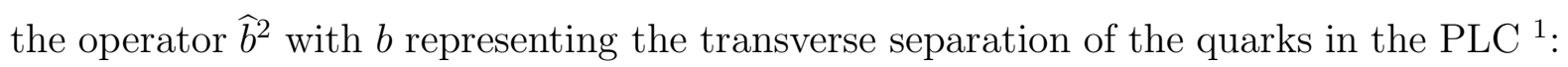

$$
b^{2}(t)=\frac{1}{c_{1}}<\pi\left|e^{\hat{H} t} \hat{b}^{2} e^{-\hat{H} t}\right| P L C>.
$$

\footnotetext{
${ }^{1}$ For the real PLC the longitudinal direction is given by the momentum transfer in the hard interaction. Unlike real PLC our wave packet is spherically symmetrical so we arbitrarily choose the $z-$ direction to be longitudinal.
} 
Here we normalize it by $c_{1}=<\pi \mid P L C>$, which represents the amplitude of the detecting the pion without the soft interaction. To find the matrix element (可) we need to know the wave functions of the PLC and the pion. There is no unique way to define the wave functions on the lattice. We use the gauge invariant formulation of equal time Bethe-Salpeter amplitude $\Psi(\mathbf{r}, t)[7]$

$$
\Psi(\mathbf{r}, t)=\sum_{\mathbf{x}}<0\left|\bar{d}(\mathbf{x}, t) \gamma_{5} U(\mathbf{x} \rightarrow \mathbf{x}+\mathbf{r}, t) u(\mathbf{x}+\mathbf{r}, t) \phi(0,0)\right| 0>.
$$

The Bethe-Salpeter amplitude in the gauge invariant formulation is known [7] to underestimate the spatial extent of the pion wave function. But it does not suffer from finite lattice size errors and is hoped to give a qualitatively correct estimate of the effects under consideration.

In a fashion similar to $G_{2}(t) \Psi(\mathbf{r}, t)$ can be expanded in a complete set of states

$$
\Psi(\mathbf{r}, t)=\sum_{\nu}\left(\int d \nu\right) c_{\nu} \psi_{\nu}(\mathbf{r}) e^{-E_{\nu} t}
$$

where

$$
\psi_{\nu}(\mathbf{r})=<0\left|\bar{d}(0,0) \gamma_{5} U(\mathbf{r}, t) u(\mathbf{r}, t)\right| \nu>/ \sqrt{2 E_{\nu}}
$$

is the Bethe-Salpeter amplitude of the state $\mid \nu>$.

$\Psi(\mathbf{r}, t)$ is measured on the lattice and $b^{2}(t)$ is calculated according to

$$
b^{2}(t)=\int d^{3} \mathbf{r} \Psi(\mathbf{r}, t) \psi_{\pi}(\mathbf{r}, t)(r \cos \theta)^{2}
$$

The ground state wave function $\psi_{\pi}(\mathbf{r}, t)$ is obtained from $\Psi(\mathbf{r}, t)$ in the limit $t \rightarrow \infty$.

$b^{2}(t)$ has the following spectral representation

$$
b^{2}(t)=\sum_{\nu}\left(\int d \nu\right) \frac{c_{\nu}}{c_{1}} b_{\nu}^{2} e^{-\left(E_{\nu}-E_{\pi}\right) t}
$$

where

$$
b_{\nu}^{2}=\int d^{3} \mathbf{r} \psi_{\nu}(\mathbf{r}, t) \psi_{\pi}(\mathbf{r})(r \cos \theta)^{2} .
$$

The spectral representation ( 10) can be used to perform the transition from the Euclidean time to the Minkowsky time. If we knew the $E_{\nu}$ 's, $c_{\nu}$ 's, and $b_{\nu}$ 's for all the 
contributing states, then a mere substitution $t \rightarrow i \tau$ would give us $b^{2}(\tau)$, which describes the expansion of the PLC in the real time. This is certainly too much to ask for. Since we work with the Euclidean time most of the states decay away too quickly to be detected.

We assume that the spectral density can be represented as a sum of several sharp poles. With this assumption the goal is to try to determine the $E_{\nu}$ 's, $c_{\nu}$ 's, and $b_{\nu}$ 's for as many states as possible. We perform the many pole fit of $b^{2}(t)$ with the fit function $f_{b}(t)$ :

$$
f_{b}(t)=\sum_{n}^{N_{p}} a_{n} e^{-E_{n} t},
$$

where $N_{p}$ is the number of the poles. However, there are several sticky points here. First, there has been some concern expressed in the literature [12] about the validity of such a many pole fit. Second, it is not a priori obvious that those few states we are able to extract will be enough to form a small or at least reduced size wave packet. In Sec. 1 and Sec. 5 we show that a simultaneous three pole fit of $b^{2}(t)$ and $G_{2}(t)$ can be reliably performed to yield the parameters for the three lowest states. These three states do form a reduced size wave packet, whose expansion is considered in Sec. 6 But first we give a summary of the lattice calculations in the next section.

\section{LATTICE DETAILS}

The calculations are performed for $S U(2)$ gauge theory with the coupling constant $\beta=$ 2.5. $S U(2)$ is chosen to increase the efficiency of the calculation and improve statistics. The size of the lattice is $12^{3} \times 24$. 60 quenched gauge field configurations are generated using Metropolis method with overrelaxation [9]. The first configuration is selected after 2000 thermalization sweeps and all the consecutive ones after 1000 sweeps.

The Wilson propagators are calculated for three values of the hopping parameter $\kappa=$ $0.146,0.148,0.149$. Periodic boundary condition in the spatial directions and Dirichlet boundary conditions in the time direction are used. A standard procedure [7] is used to construct the two-point correlation function and the Bathe-Salpeter amplitude out of 
the quark propagators.

The lattice spacing is determined from the $\rho$ mass [8] to be $a=0.09 \pm 0.012 \mathrm{fm}$, with is in quantitive agreement with the results [4 obtained from the string tension.

The quark mass $m_{q}$ is conventionally associated with the hopping parameter $\kappa: m_{q}=$ $\left(1 / 2 \kappa-1 / 2 \kappa_{c r}\right) a^{-1}$. Extrapolation to the limit $m_{\pi}=0$ yields $\kappa_{c r}=0.151(1)$. With this $\kappa_{c r}$ we obtain three values of the quark mass $m_{q}=275,174,124 \mathrm{Mev}$.

The covariance matrix fit is performed to determine the parameters needed. A detailed description of this procedure is given in Sec. 4. All statistical errors are estimated by the single elimination jacknife [10]. The point source is placed at the time slice $t=5$ and the fitting is performed over the range $t=6$ through $t=20$.

\section{MANY POLE FIT}

A many pole fit involves many problems, some of which are mentioned in [12, 11]. To address these problems we perform a detailed analysis of the fit of the two-point correlation function $G_{2}(t)$. The fit function $f_{G}(t)$

$$
f_{G}(t)=\sum_{n=1}^{N_{p}} c_{n} e^{-E_{n} t}
$$

corresponds to $N_{p}$-pole fit.

We would like to emphasize that our goal here is to extract as many states as possible to be able to form a small size wave packet. This is contrary to the intention of the majority of the papers in the field, where the isolation of one state, usually the ground state, is pursued.

A standard approach in determining parameters $c_{n}$ 's and $E_{n}$ 's is to minimize the goodness-of-fit statistic $\chi^{2}$.

$$
\chi^{2}=\sum_{t, t^{\prime}}\left(G_{2}(t)-f_{G}(t)\right) C\left(t, t^{\prime}\right)^{-1}\left(G_{2}\left(t^{\prime}\right)-f_{G}\left(t^{\prime}\right)\right.
$$


The jacknife method is used to calculate the covariance matrix $C\left(t, t^{\prime}\right)$

$$
C\left(t, t^{\prime}\right)=\frac{1}{N_{c}-1} \sum_{k=1}^{N_{c}}\left(G_{2}^{[k]}(t)-G_{2}(t)\right)\left(G_{2}^{[k]}\left(t^{\prime}\right)-G_{2}\left(t^{\prime}\right)\right.
$$

where $N_{c}$ is the number of configurations, $G_{2}^{[k]}(t)$ are found for a subset of $N_{c}-1$ configurations with $k^{\prime}$ th configurations omitted.

Simulated annealing is our choice of the minimization technique. The full covariance matrix is used in the minimization(correlated $\chi^{2}$ fit). There have been studies [16, 17] to show that for a small data sample use of the full covariance matrix is unreliable. In [11] a use of several well defined eigenmodes of the covariant matrix is proposed. The use of a full covariant matrix versus the diagonal part thereof poses an interesting problem. We address this problem in the Appendix, where we consider a simple example amenable to the analytical treatment. The conclusion is reached that a correlated fit gives better results if the correlations are appreciable. This is generally the case for lattice calculations, and it is true for our calculations. Moreover, we consider 60 configurations to be sufficient for the maximum of 9 degrees of freedom we have while fitting $G_{2}(t)$.

The fitting is performed over a time range extending from $t_{\text {first }}$ to $t_{\text {last }}$. We choose $t_{\text {last }}=20$ to exclude the boundary effects and $t_{\text {first }}$ is varied from 6 to 14 . The number of states used in a fit is determined by the $t_{f i r s t}$. One of the problems with a many pole fit [12] is that the result depends on the $t_{\text {first }}$ used for the fit, and it is not a priori clear how many states should be included in a fit.

The strategy developed to tackle this problem is as follows. We start with $t_{\text {first }}$ for which only the ground state contribution is significant, and one-pole fit is performed. As $t_{\text {first }}$ decreases more states with higher masses come into play. To decide between two fits with different number of poles for a particular $t_{\text {first }}$ the preference is given to a fit with smaller value of $\chi^{2}$ per degree of freedom. The results for the $E_{n}$ 's for the hopping parameter $\kappa=0.149$ are shown in Fig. 1 . For $t_{\text {first }}=12 \div 14$ one state is enough, for $t_{\text {first }}=9 \div 11$ two states had to be included, and for $t_{\text {first }}=6 \div 8$ the $G_{2}(t)$ is best fit with three states. The results for the other two hopping parameters are similar. The 
values of the $\chi^{2}$ per degree of freedom show that the fit for the longest time range is the best ( Table 1).

The pattern in the results for $E_{n}$ is obvious. The statistical error is the greatest when a new state is included in the fit, it then decreases and jumps back, when another state is included. To explain this pattern the following plot is made. The individual contributions $a_{n} e^{-E_{n} t}$ of the states to the two-point correlation function $G_{2}(t)$ are shown in Fig. 2. They are compared with the statistical error on the $G_{2}(t)$. Two time slices $t_{\text {first }}=12$ and $t_{\text {first }}=9$ are important, since at those times the second and third states, go above the statistical noise. Those are precisely the time slices for which the contribution of these states is included in the fit.

A simple rule follows: a state should be included if its contribution is greater than the statistical error; it should not be included if its contribution is less than the statistical error. The best results of the fitting are obtained for the longest time span of the highest energy state used in the fitting. An interesting consequence is that, in general, the longest time range is not necessarily the best for determining the parameters. Consider the following example. Suppose that in Fig. 1 the results of the three pole fit started at $t_{\text {first }}=8$ (i.e. the source was at $t=7$ and the results for $t_{\text {first }}=6$ and 7 were not present). In that case a reliable determination of the third state's parameters would be impossible; furthermore we would be forced to use the shorter time range with $t_{\text {first }}=9$ to determine the parameters of the two lowest states.

Another interesting observation concerns the fact that $\chi^{2}$ has many minima in the multi-dimensional space of $c_{n}$ 's and $E_{n}$ 's. The "real" minimum is not always the global one. By the "real" we mean the one we believe to be the best fit. In our case the best fit is obtained for the $t_{\text {first }}=6$. We ran the minimization for $t_{\text {first }}=7$ and $t_{\text {first }}=8$ and found local minima very close to the "real" one. The gain in $\chi^{2}$ compared to the global minimum is only $\approx 0.1$ per degree of freedom.

To support this argument the following simulation is performed. We work our way 
backwards. Four sets of $\tilde{c}_{n}$ and $\tilde{E}_{n}$ are chosen. A "correlation function" $\tilde{G}(t)$ is calculated:

$$
\tilde{G}(t)=\sum_{n=1,4} \tilde{c}_{n} \exp \left(-\tilde{E}_{n} t\right)
$$

Then statistical noise is added and 60 "configurations" are generated. The subsequent fitting procedure yield results that have all the basic features of the fitting of the lattice data(Fig. 3). The input values of the parameters of three states are recovered (within the error bars). The fourth state is intentionally chosen to decay below the statistical noise by $t=6$. In this simulation we know what the correct values of the parameters are, and we can verify that for $t_{\text {first }}=7$ and $t_{\text {first }}=8$ there are local minima very close to these correct values.

We conclude that for the given choice of interpolating field and lattice spacing three pole fit of the two-point correlation function can be performed and the values of the excitation strength $c_{n}$ and the mass $E_{n}$ reliably determined. These values are presented in Table 2.

\section{$5 b^{2}(t)$ MANY POLE FIT}

The determination of the $c_{n}$ 's and $E_{n}$ 's is interesting, but it is does not constitute the main objective of this paper. What does is the strength of the soft interaction $b^{2}(t)$, which we fit with the Eq.( 12). As was indicated in the introduction, a fit of $b^{2}(t)$ alone does not give a satisfactory result. To understand it, note that a coefficient $a_{n}$ in Eq.( 12) is proportional to the excitation strength $c_{n}$ and the soft interaction transition matrix element $b_{n}^{2}$ of the state $|n\rangle$. For the three states, that we are able to extract from the two-point correlation function $G_{2}(t)$, the excitation strength $c_{n}$ grows with $n$. (Physically it means that their wave functions have a better overlap with the wave function of the initially formed wave packet.) Unlike the coefficients $c_{n} b_{n}^{2}$ turned out to decrease rapidly with $n$. As a consequence the contribution to the $b^{2}(t)$ of the exited states disappears below the statistical noise much sooner than for the $G_{2}(t)$. This forces us to fit $b^{2}(t)$ together with $G_{2}(t)$. 
We would like to note here that the procedure we use to compute $b^{2}(t)$ is akin to the smearing technique used in [13, 14] to isolate the contribution of meson and baryon ground states. However, let us stress it again, this is not our intention. This is an "accident", but an "accident" with some physical consequences, which are discussed below.

The simultaneous fit of $b^{2}(t)$ and $G_{2}(t)$ is performed for the longest time range possible $\left(t_{\text {first }}=6\right)$. The results are shown in the Table $3 . b_{n}^{2}$ goes down by a factor of 2 with

every excited state. Another important feature is that $b_{3}^{2}$ is negative. This allows for the formation of a reduced size wave packet.

Let's discuss the physical meaning of these results. Do they have any relevance for color transparency? Again consider (e,e'p) process. In this case the coefficients $c_{\nu}$ defining the PLC are the elastic and inelastic form factors of the ground state. In general we can expect these coefficients to exibit a resonant behavior - to have a peak for a certain excited state. But the soft interaction supresses the contribution of the higher excited states, as $b_{\nu}$ decreases rapidly with $\nu[15]$. This suppression plays a crucial role in achieving a slow expansion rate of the PLC. Our result turned out to have these basic features. The $c_{n}$ grows with $n$, whereas $b_{n}$ decreases rapidly. The former is only a result of our choice of the model wave packet and in this sense is arbitrary. The latter, however, reflects the properties of hadronic states in lattice QCD, and to the extent that we believe lattice QCD, this is an indication of the possibility of the suppression of the contribution of the higher excited states by the soft interaction.

\section{MINKOWSKY TIME EXPANSION}

Having obtained the parameters $E_{n}, c_{n}, b_{n}^{2}$ for the three lowest states, we substitute $t \rightarrow i \tau$ in Eq. (10) and plot the real part of $b^{2}(\tau)$ (Fig. 4).

$$
\operatorname{Re}\left(b^{2}(\tau)\right)=\sum_{n=1}^{3} \frac{c_{n}}{c_{1}} b_{n}^{2} \cos \left(E_{n}-E_{1}\right) \tau
$$

The band shown in Fig. 4 corresponds to the 60 sets of 59 configurations used in the jacknife evaluation. Each of the curves shows an expansion. We do not compute a set of 
curves simply using the errors quoted in Table 2 and Table 3. Such a procedure would incorrectly ignore the correlations between the parameters $E_{n}, c_{n}$, and $b_{n}^{2}$.

A look at Fig. 4 shows us that the wave packet under investigation is not really small. According to Fig. $4, b^{2}(0) \approx 3 / 4 b_{1}^{2}$, where $b_{1}^{2}=<\pi\left|b^{2}\right| \pi>$ is the ground state contibution. (In the Fig. $4 b_{1}^{2}$ is represented by the solid horizontal line.) This is because we could recover only three out of the many states comprising the wave packet at the source $t=5$. Furthermore, even at $t=5$ the wave packet is not quite point like. As was mentioned above, there is a non-zero probability for the quark and the antiquark to be separated by some distance at the time they are created by the point interpolating field.

These features make it difficult to define one number $\tau_{\text {exp }}$ to typify the time for the wave packet to expand. One way is to define $\tau_{\text {exp }}$ as the time it takes for $b^{2}(\tau)$ to reach the ground state contribution $b_{1}^{2}$. This expansion time is found to be $\approx 0.03 \mathrm{fm}$, which is certainly much smaller than the root mean square radius of the pion $\approx 0.4 \mathrm{fm}$ here 2. However, this estimate is misleading, since the initial wave packet is not small. The initial wave packet size is determined by the coefficients $c_{n}$ as well as $b_{n}^{2}$. Here the set $c_{n}$ determines the spectral density of the operator $\phi(x)$. These numbers are only related to color transparency physics if they also represent the elastic and inelastic form factors. Thus we are free to consider the consequences of using different values of $c_{n}$. Varying $c_{n}$ we can obtain an upper limit for the expansion time for the given energies $E_{n}$ and soft interaction matrix elements $b_{n}^{2}$. This is achieved for $c_{3}=0$ and $c_{2}<0$ and equal to $\tau_{\text {exp }} \approx 2 \mathrm{fm}$.

The results for the other two hopping parameters are similar to the one shown in Fig. 4, but with bigger statistical error. For the purpose of our investigation we are not interested in taking the physical limit $E_{1} \rightarrow m_{\pi}$. The point is that the expansion time is determined by the energy differences $E_{n}-E_{1}$ as well as $c_{n}$ and $b_{n}^{2}$. The coefficients $c_{n}$ and $b_{n}^{2}$ are virtually independent of the quark mass. The lowest value of the energy gap

\footnotetext{
${ }^{2}$ This small number is the consequence of using the gauge invariant formulation of the Bethe-Salpeter wave function.
} 
for a pion is much larger, than for a proton. By taking the physical limit to $m_{\pi} \approx 0$ we only increase the mass gap and decrease the expansion time.

There is one more point to be made. The subject of our investigation is the expansion of a wave packet in its rest frame. If color transparency is to be observed, it will be in a high momentum transfer reaction, when the escaping wave packet is moving with a speed close to the speed of light. Time dilation will increase the expansion time. In fact, color transparency can be achieved for any small size wave packet, consisting of a finite number of states, as long as the momentum transfer is much greater than the energy of the states. A real PLC produced in a high momentum transfer reaction consists of an infinite number of states. How the energy of the important states changes with the momentum transfer will decide the fate of color transparency.

\section{CONCLUSION}

The expansion of a reduced size wave packet on the lattice is investigated. The wave packet is created with the pseudoscalar point interpolating field. The quantity representing the strength of the soft interaction of the expanding wave packet with the nucleus is measured as a function of the Euclidean time. We treat the expanding wave packet as a coherent sum of physical states. Most of the states decay away before we can detect them. The three lowest energy states we are able to recover allow for the formation of a reduced size wave packet. With the parameters of these states we are able to do the transition to the Minkowsky time. A Minkowsky time expansion picture is obtained.

What is the importance of our work and where do we go from here? For the first time three states - a ground state and two excited states- are obtained from the lattice calculations. (One may attempt to recover more excited states by using a smaller lattice spacing a.) The matrix elements of the soft interaction between the ground state and the two excited states are important for color transparency. These are found to decay rapidly with energy. This feature is an important component of color transparency. (It 
would be useful to verify that similar results are obtained for baryons.) Furthermore, the present results show that a lattice evaluation of wave packet expansion is possible.

In the present results, the initial wave packet is not small, and its relation to color transparency physics is unclear. Our next step will be to attack this problem by studying the wave packet (hopefully a PLC) created by the electromagnetic current operator.

It is our pleasure to thank Steven Sharpe for useful discussions and encouragement. This work was supported in part by the U.S. Department of Energy. The lattice calculations were performed on the UW Nuclear Theory DECstation 3000-600 AXP.

\section{A FULL VS. DIAGONAL COVARIANCE MATRIX FIT}

There have been several papers, in which the attention has been brought to the fact that the use of the full covariance matrix for the correlated data is unreliable for small data samples [16, 17]. We use a simple example to show analytically that there are instances, when the use of the diagonal part of a covariance matrix to fit data (uncorrelated $\chi^{2}$ fit) with a theoretical expression gives a better result than the full covariance matrix

(correlated $\chi^{2}$ fit), even in the limit of infinite number of samples. Note that when we use the diagonal part, we fit correlated data and simply ignore the correlations among them.

Consider fitting results of the $N_{\text {sample }}$ measurements of $N$ quantities $x$ by a constant I. We assume that all $\overline{x_{i}}$ 's

$$
\overline{x_{i}}=\frac{1}{N_{\text {sample }}} \sum_{\alpha=1}^{N_{\text {sample }}} x_{i}^{\alpha}
$$

do reach the same constant value $J$ in the limit $N_{\text {sample }} \rightarrow \infty$ and that the correct infinite $N_{\text {sample }}$ form of the covariance matrix $C_{i k}$ is known. 
Then we can minimize $\chi^{2}$,

$$
\chi^{2}=\sum_{i k}\left(\overline{x_{i}}-I\right) C_{i k}\left(\overline{x_{k}}-I\right)
$$

with respect to $I$ and find $I$ in two cases: $I_{\text {full }}$, when the full covariance matrix $C$ is used, and $I_{\text {diag }}$, when only the diagonal elements of the covariance matrix $C$ are used.

$$
I_{\text {full }}=\frac{\sum_{i k} C_{i k}^{-1} \overline{x_{k}}}{\sum_{i k} C_{i k}^{-1}}, \quad I_{\text {diag }}=\frac{\sum_{i} \overline{x_{i}} / C_{i i}}{\sum_{i} 1 / C_{i i}}
$$

Both $I_{\text {full }}$ and $I_{d i a g}$ are unbiased estimators of the true value $J$, since in the limit $N_{\text {sample }} \rightarrow \infty$ they both are equal to $J$. The preference should be given to the estimator with the smaller standard deviation. Results for $I_{\text {full }}$ and $I_{\text {diag }}$ will vary from one set of $N_{\text {sample }}$ samples to another with the variance $C_{I}=\overline{I^{2}}-(\bar{I})^{2}$ equal to

$$
C_{I_{f u l l}}=\left(\sum_{i k} C_{i k}^{-1}\right)^{-1} \quad C_{I_{d i a g}}=\left(\sum_{i} \frac{1}{C_{i i}}\right)^{-1} .
$$

From this general form it is clear that we can not expect $C_{I_{f u l l}}<C_{I_{\text {diag }}}$ to be always true. Consider a situation, when the diagonal elements of the matrix $C$ are much greater than nondiagonal:

$$
\frac{C_{i k}}{C_{i i}} \ll 1 ; \quad \frac{C_{k k} C_{i k}^{2}}{C_{i i}} \ll 1 ; \quad i \neq k
$$

and we can expand $C^{-1}$ to get for $C_{I_{f u l l}}$

$$
C_{I_{\text {full }}}=\left(\sum_{i} \frac{1}{C_{i i}}-\sum_{i \neq k} \frac{C_{i i}}{C_{k k}} C_{i k}\right)^{-1}
$$

The uncorrelated fit yields better results in the case of prevailing positive correlations, the correlated fit is better in the case of prevailing negative correlations. But positive correlations do not necessarily render a correlated fit worse than uncorrelated one. Consider a simple example of $N=2$, when the expressions for $C_{I_{\text {full }}}$ and $C_{I_{\text {diag }}}$ become transparent:

$$
C_{I_{\text {full }}}=\frac{C_{11} C_{22}-C_{12}^{2}}{C_{11}+C_{22}-2 C_{12}} \quad C_{I_{\text {diag }}}=\frac{C_{11} C_{22}}{C_{11}+C_{22}}
$$


Again, as in the case of small correlations, a correlated fit is better for negative correlations $C_{12}<0$. But there is a region of big positive correlations

$$
C_{12}>\frac{2 C_{11} C_{22}}{C_{11}+C_{22}}
$$

where a correlated fit gives better results.

Another issue is using $\chi^{2}$ to estimate how good a fit is. For the correlated fit the infinite $N_{\text {sample }}$ limit of $\chi_{\text {full }}^{2}$ is $N-1$, which is exactly 1 per degree of freedom. In the case of the uncorrelated fit $\chi^{2}$ reaches this limit only if the data is genuinely uncorrelated $\left(C_{i k}=0, i \neq k\right)$. When we ignore the correlations and use the diagonal part of the correlation matrix to obtain the result for $I$, they pop up in the expression for $\chi_{\text {diag }}^{2}$ :

$$
\chi_{\text {diag }}^{2}=N-1-\sum_{i \neq k} \frac{C_{i k}}{C_{i i} C_{k k}} / \sum_{i}\left(C_{i i}\right)^{-1}
$$

One therefore can not always use $\chi^{2}$ to evaluate an uncorrelated fit.

\section{References}

[1] L. Frankfurt, G.A. Miller, and M. Strikman, Ann. Rev. Nucl. Part. Sci. 45, (1994) 501

[2] S.J. Brodsky, in Proc. Thirteenth Int. Symp. on multiparticle dynamics, ed.W. Kittel, W. Metzger, and A. Stergiou (World Scientific, Singapore 1982) p.963

[3] A.H. Mueller in Proceedings of Seventeenth rencontre de Moriond, Moriond, 1982 ed. J Tran Thanh Van (Editions Frontieres, Gif-surr-Yvette, France, 1982) Vol. I, p13.

[4] A.M. Green, C. Michael, J. Paton and M.E. Sainio, Int. J. Mod. Phys. E2, (1993) 479.

[5] G.A. Miller, Int. J. of Mod. Phys. E1, (1992) 629. 


\begin{tabular}{|c|c|c|c|c|c|c|c|c|c|}
\hline$t_{\text {first }}$ & 6 & 7 & 8 & 9 & 10 & 11 & 12 & 13 & 14 \\
\hline$\chi^{2}$ & 1 & 1.2 & 1.6 & 1.5 & 1.8 & 2 & 2.3 & 1.6 & 0.3 \\
\hline
\end{tabular}

Table 1: $\chi^{2}$ per degree of freedom for different time ranges

[6] D. Toussaint's TASI lectures in From Action to Answers (World Scientific, 1990) p.121

[7] M.-C. Chu, M. Lissia, J.W. Negele, Nucl.Phys. B360, (1991) 31.

[8] G. Martinelli, C.T. Sachrajda, Nucl. Phys. B306, (1988) 865.

[9] R.Gupta, G.W. Kilcup, A. Patel, S.R. Sharpe, P. de Forcrand, Mod. Phys. Lett., A3, (1988) 1367.

[10] B. Efron, SIAM Rev, 21, (1979) 460.

[11] G.W. Kilcup, Nucl. Phys. B (Proc. Suppl.) 34, (1994) 350.

[12] D.B. Leinweber, OSU preprint 94-0332 (1994), submitted to Phys. Rev. D.

[13] P. Bacilier et. al., Nucl. Phys. B317, (1989) 509.

[14] A. Duncan, E. Eichten, H. Thacker, Nucl. Phys. B (Proc. Suppl.) 26, (1992) 394.

[15] D.Makovoz and G.A.Miller, UW preprint 40427-25-N94, submitted to Phys. Rev. C.

[16] C.Michael, Liverpool preprint LTH 321, hep-lat/9310026.

[17] D.Seibert, CERN preprint TH 6892 (1993), submitted to Phys. Rev. D. 


\begin{tabular}{llll}
\hline \hline & & & \\
Parameter & $\kappa_{1}=0.146$ & $\kappa_{2}=0.148$ & $\kappa_{3}=0.149$ \\
& & & \\
$E_{1} a$ & $0.49(1)$ & $0.39(4)$ & $0.33(5)$ \\
$E_{2} a$ & $1.2(1)$ & $1.1(2)$ & $1.0(2)$ \\
$E_{3} a$ & & & \\
& $2.2(1)$ & $2.2(2)$ & $0.35(6)$ \\
$c_{1}$ & & & \\
& $0.39(3)$ & $0.36(6)$ & $1.0(3)$ \\
$c_{2}$ & & & $2.4(6)$ \\
$c_{3}$ & $1.1(2)$ & $1.1(2)$ & \\
\hline \hline
\end{tabular}

Table 2: Energy $E_{n}$ and excitation strength $c_{n}$ of the three pole fit of the two-point correlation function $G_{2}(t)$ 
Figure 1: The energies of three states as a function of the first time slice $t_{\text {first }}$ used in the fitting. $t_{\text {last }}=20$ for all the fits. The dash line shows the result for the longest time range. Hopping parameter $\kappa=0.149$. 
Figure 2: Individual contribution of the three states to the two-point correlation function to be compared to the statistical error of the two-point correlation function $G_{2}(t)$. Hopping parameter $\kappa=0.149$. 
Figure 3: The energies of three states for the simulated $\tilde{G}(t) . t_{l a s t}=20$ for all the fits. The dash lines show the correct input values of the energies. 
Figure 4: Expansion of the wave packet in real time. The solid curve corresponds to the average over all 60 configurations. The dash curves are obtained for 60 sets of 59 configurations that were used in the jacknife evaluation. The horisontal solid line is the ground state contribution $b_{1}^{2}$. Hopping parameter $\beta=0.146$. 


\begin{tabular}{llll}
\hline \hline & & & \\
Parameter & $\kappa_{1}=0.146$ & $\kappa_{2}=0.148$ & $\kappa_{3}=0.149$ \\
\hline$b_{1}^{2}$ & $0.08(2)$ & $0.08(2)$ & $0.08(2)$ \\
$b_{2}^{2}$ & $0.04(1)$ & $0.05(2)$ & $0.05(2)$ \\
$b_{3}^{2}$ & & $-0.015(13)$ \\
\hline \hline
\end{tabular}

Table 3: The soft interaction matrix elements $b_{n}=<n\left|b^{2}\right| 1>$. 
This figure "fig1-1.png" is available in "png" format from: http://arxiv.org/ps/hep-lat/9411030v2 


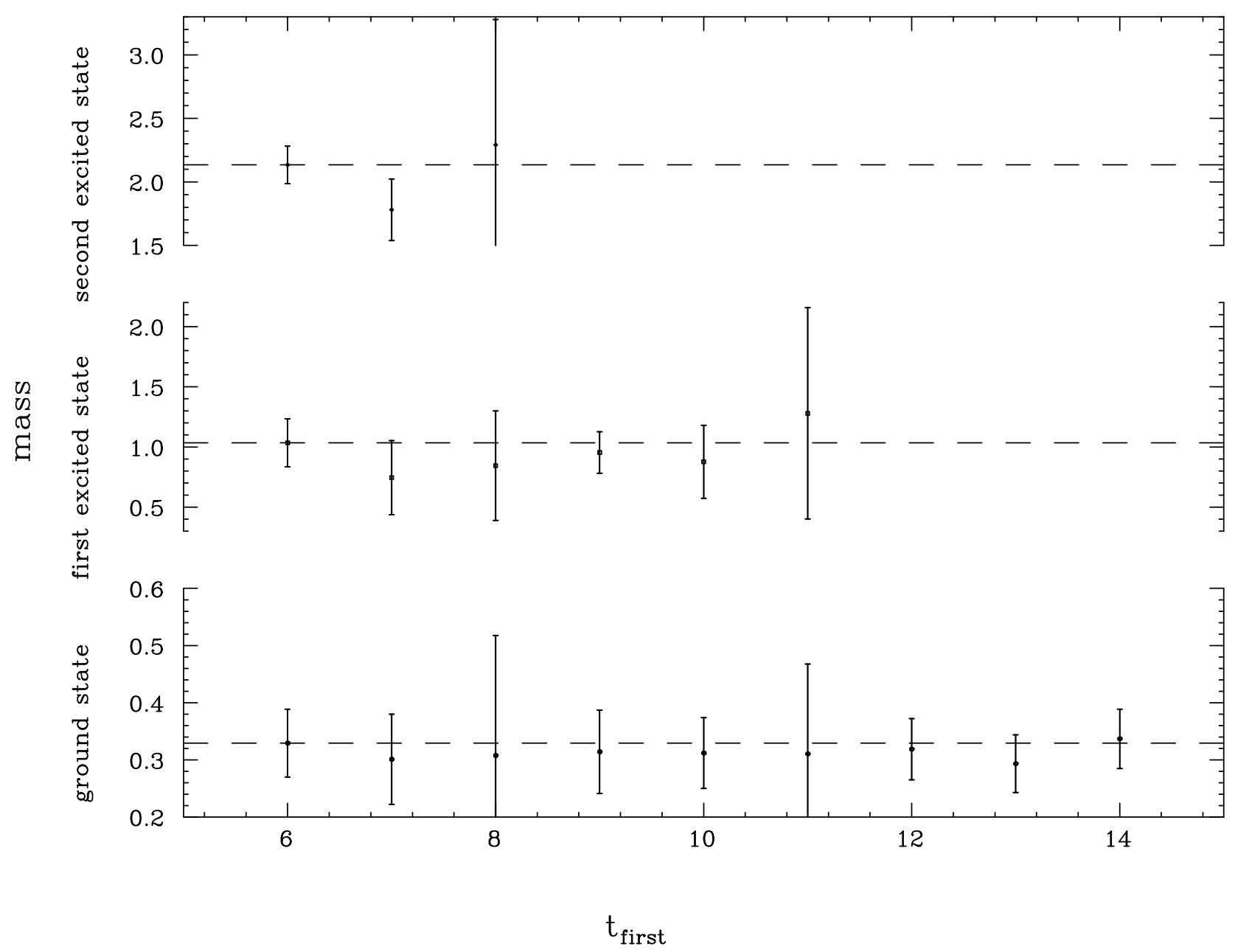


This figure "fig1-2.png" is available in "png" format from: http://arxiv.org/ps/hep-lat/9411030v2 


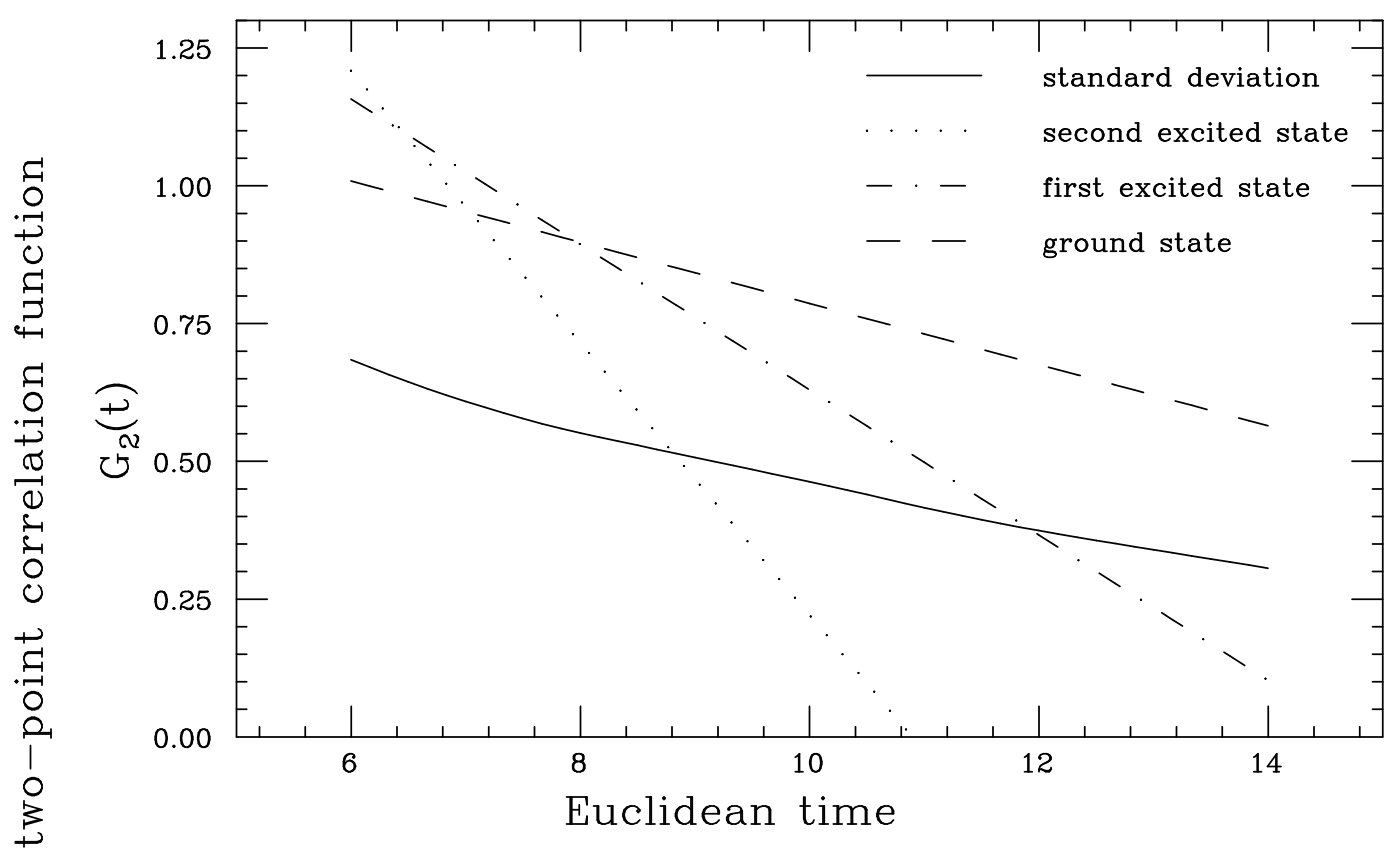


This figure "fig1-3.png" is available in "png" format from: http://arxiv.org/ps/hep-lat/9411030v2 


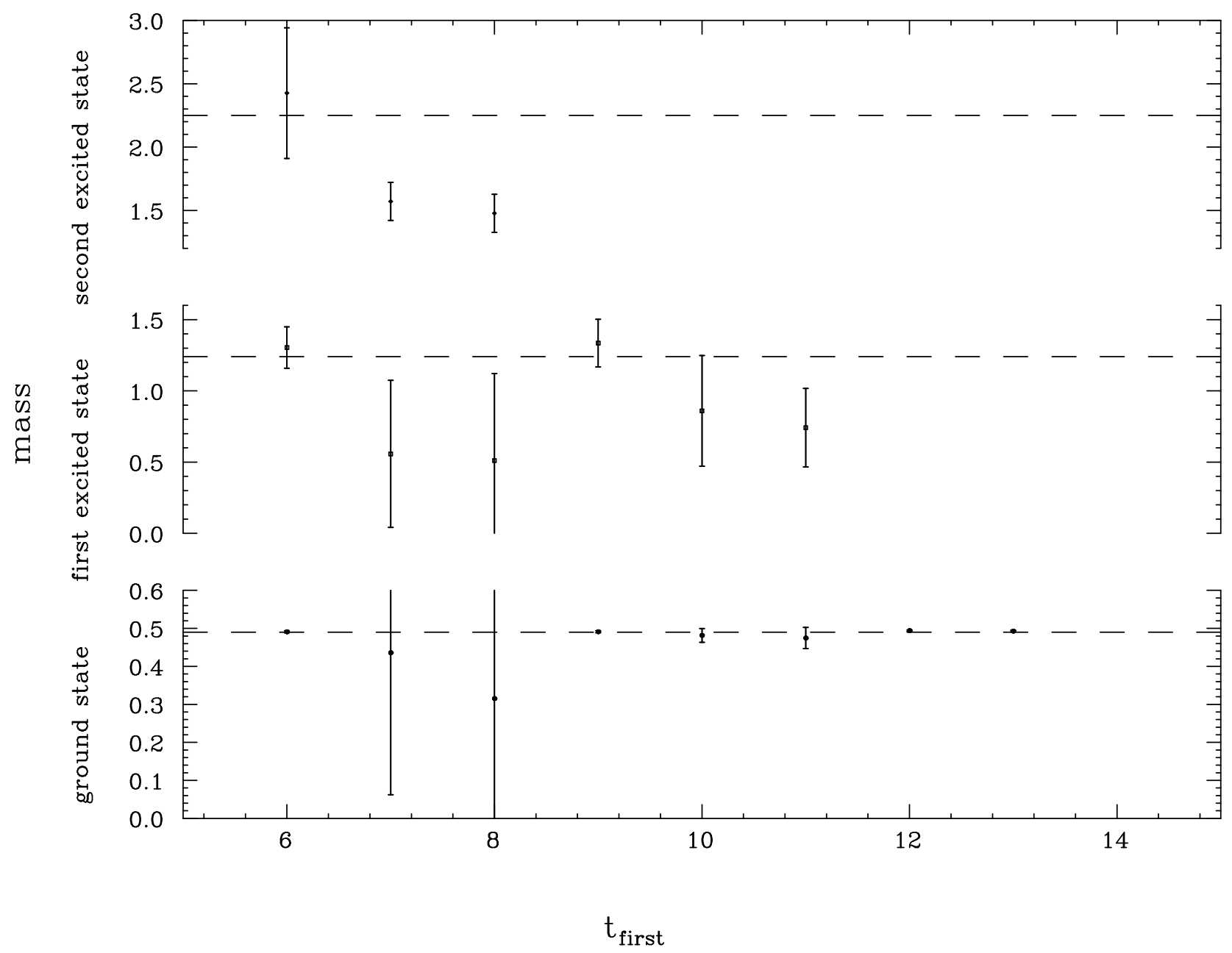


This figure "fig1-4.png" is available in "png" format from: http://arxiv.org/ps/hep-lat/9411030v2 


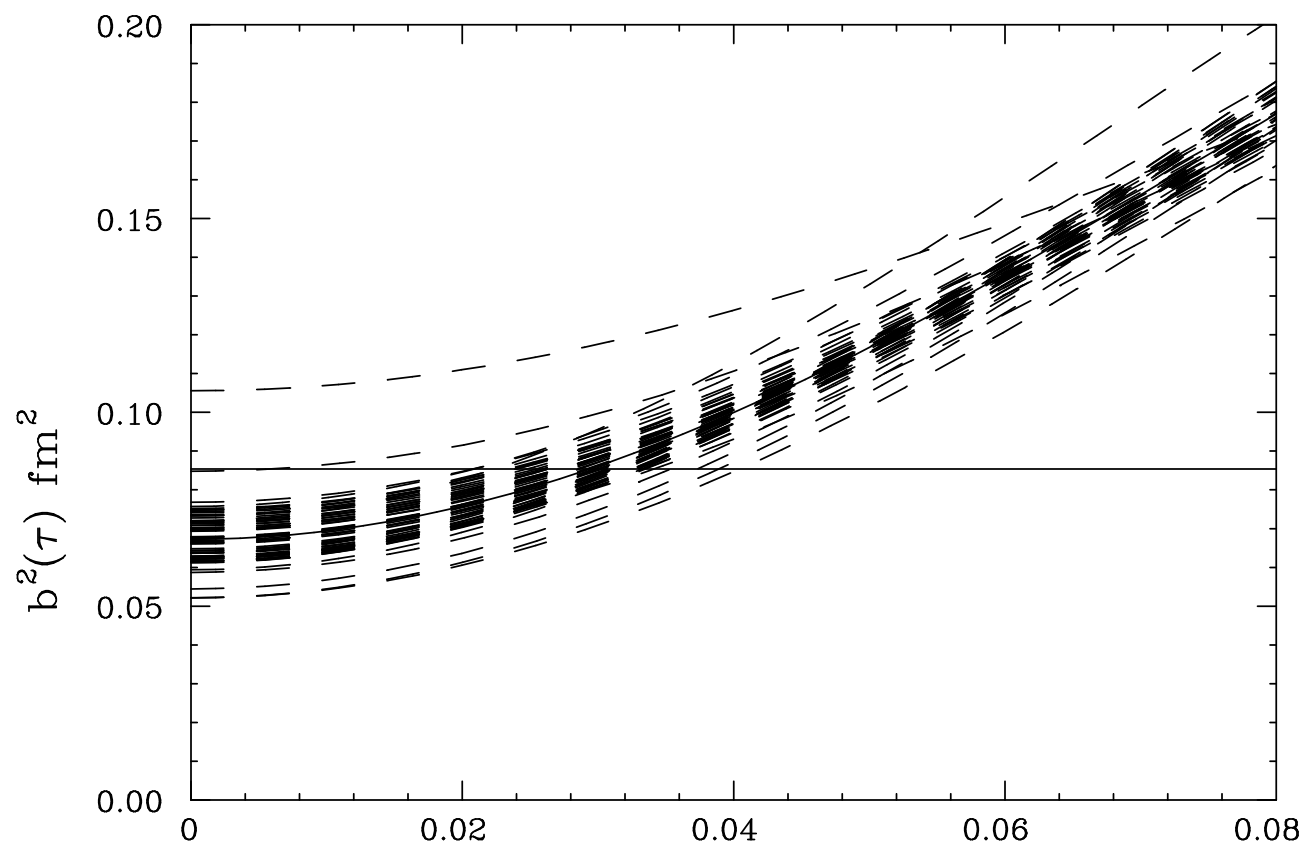

Minkowsky time $\tau$ fm 